\title{
Résistance de la luzerne (Medicago sativa L.) à des isolats de Colletotrichum trifolii (Bain et Essary) du bassin méditerranéen, notamment du Maroc
} \author{
F.-Z. Mesbah ${ }^{1}$ et F. Ferrari ${ }^{2}$ \\ 1 Université Mohammed fer, Faculté des Sciences, Département de Biologie, Oujda, Maroc \\ 2 Laboratoire de Pathologie végétale, INA-PG - INRA, 78850 Thiverval-Grignon, France \\ 3 Laboratoire d'Agronomie, INA-PG - INRA, 78850 Thiverval-Grignon, France
}

H. Gosset ${ }^{1}$, B.M. Trœung ${ }^{1}$, G. Raynal ${ }^{2}$, P. Huet ${ }^{3}$ avec la collaboration technique de N. Boukhari ${ }^{1}$,

(reçu le 7 juin 1988, accepté le 16 février 1989)

Résumé - Colletotrichum trifolii, agent de l'anthracnose de la luzerne, est important sur cette culture dans le Maroc oriental. Des contaminations artificielles effectuées à l'aide de 2 isolats de la région d'Oujda sur 22 cultivars et lignées de luzerne d'origine nord-américaine, flamande, méditerranéenne et marocaine, montrent que seul le cultivar américain Arc de même que 2 autres lignées américaines et une lignée française sont très résistants. Les autres cultivars ont des degrés de sensibilité divers. Certains présentent 10 à $15 \%$ de plantes résistantes (Gemini, Vertus), mais la majorité sont sensibles à très sensibles. La comparaison du pouvoir pathogène de 5 isolats du Maroc, du bassin méditerranéen et du Nord de la France, montre qu'ils sont très voisins par leur niveau d'agressivité et qu'ils appartiennent vraisemblablement à la race 1, commune dans le monde, se caractérisant par son absence de virulence particulière.

anthracnose - Colletotrichum trifolii - tests variétaux - luzerne - pathogénie

\begin{abstract}
Summary - Resistance of alfalfa (Medicago sativa L.) to isolates of Colletotrichum trifolii (Bain et Essary) from the mediterranean basin, especially from Morocco. Colletotrichum trifolii, the agent which is responsible for alfalfa anthracnose, has a significant effect on this crop in eastern Morocco. Artificial contamination carried out by 2 isolates from the Oujda region on 22 alfalfa cultivars and breeding lines of north american, flemish, mediterranean and moroccan origin, showed that the cultivar Arc, as well as 2 american and 1 french lines are very resistant. The other cultivars showed various degrees of susceptibility. Some exhibited 10-15\% resistant plants (Gemini, Vertus), but the majority were susceptible to very susceptible. When comparing the pathogenicity between 5 isolates from Morocco, mediterranean basin and North of France, it was shown that their aggressiveness did not vary greatly and that they might belong to race 1, common throughout the world, and characterised by its lack of special virulence.
\end{abstract}

anthracnose - Colletotrichum trifolii - varietal tests - alfalfa - pathogeny

\section{Introduction}

Depuis que Bain et Essary (1906) imputèrent pour la première fois des cas d'anthracnose de la luzerne à Colletotrichum trifolii, nombre d'auteurs ont signalé les dégâts dont ce champignon est responsable. Aux Etats-Unis, par exemple, Barnes et al. (1969) considéraient que l'attaque par $C$. trifolii pouvait réduire le rendement en fourrage dans les régions chaudes et humides dans des proportions allant de 25 à $30 \%$. Dans ce même pays, Elgin et al. (1981) ont prouvé que l'introduction de cultivars résistants permettait une augmentation moyenne de la production de l'ordre de $10 \%$ et, dans les meilleurs cas, de près de $20 \%$, principalement dans les Etats du nordest, à climat favorable à la maladie.

Lors de nos premières observations dans le Maroc oriental, en 1986, nous avons observé 700 tiges prélevées au hasard sur une parcelle de 2 ha. C'était en automne, période la plus propice au développement du parasite dans la région considérée. Sur la variété Moapa, la plus cultivée dans le Maroc oriental, $43 \%$ des tiges présentaient des nécroses de longueur au moins égale à $3 \mathrm{~mm}$. Parmi elles, 17\% étaient, ou bien 
déjà mortes, ou bien en voie de dessèchement et porteuses d'acervules nombreuses de Colletotrichum trifolii (Trœung et Gosset, 1987). II est permis de penser qu'à long terme des pieds aussi fortement attaqués meurent ou soient incapables d'engendrer de nouvelles pousses en quantité suffisante, d'où une baisse considérable de la production fourragère.

La lutte chimique n'étant pas, jusqu'à ce jour, envisagée en culture fourragère, nous avons entrepris l'évaluation de la résistance de différents cultivars et lignées de Medicago sativa $\mathrm{L}$. éventuellement utilisables au Maroc ou intéressants, possédant des caractères connus de résistance ou de sensibilité à d'autres isolats. Pour effectuer les tests, nous avons utilisé des isolats marocains, français, portugais et yougoslaves, afin, en particulier, de vérifier s'il existe ou non des différences de pouvoir pathogène selon l'origine géographique des isolats.

\section{Matériel et Méthodes}

Les expérimentations ont été menées parallèlement au laboratoire de Pathologie végétale de Grignon de l'Institut National Agronomique Paris-Grignon et au Département de Biologie de l'Université d'Oujda, selon des méthodes assez semblables.

\section{Matériel biologique}

\section{Cultivars de luzerne et obtention des plantes}

Vingt-deux cultivars ou lignées de $M$. sativa ont été testés :

- 8 d'origine Nord-américaine (Arc, MSA CW3 An3, Beltsville 2An4, Moapa, UC-Salton, Sonora, WL 514, WL 515). Remarquons que les semences de Moapa que nous avons utilisées sont celles commercialisées au Maroc. Elles sont, pour la plupart, importées d'Australie où elles sont multipliées et ont pour origine une Moapa sélectionnée en 1963 aux Etats-Unis. Les semences dont nous disposions provenaient sans doute de lots différents, ce qui peut expliquer une certaine hétérogénéité dans les résultats obtenus avec ce cultivar. Signalons enfin que Moapa, provenant d'une population égyptienne, appartient au type méditerranéen;

- 7 d'origine européenne : 3 de type flamand (Vertus, Gémini, Europe), et 4 de type méditerranéen (Magali, Mireille, MAM M1 C3, MAP C3);

- 5 écotypes marocains de type présaharien (Demnate 1, Demnate 4, Ziz 7, Ziz 8 et Ziz 10);

-2 d'origine australienne (CAB 77-78, Siriver).

A titre de comparaison, nous avons également testé une luzerne sauvage, $M$. truncatula Gaertn.

Les plantules de 3 jours, issues de semences désinfectées et prégermées, sont réparties en 2 ou 4 lots de 50 et repiquées dans des terrines de $900 \mathrm{~cm}^{2}$ à Grignon, de $200 \mathrm{~cm}^{2}$ à Oujda, d'où une densité différente. Le substrat utilisé est stérilisé par la chaleur sèche. II est constitué à Grignon par un mélange terre + sable $(2 / 3,1 / 3)$ et à Oujda par du sable siliceux non traité dont chaque terrine reçoit $600 \mathrm{ml}$ de solution nutritive selon la formule d'Arnon et Hoagland (1940).

\section{Inoculum de Colletotrichum trifolii}

Les isolats marocains, au nombre de 2, ont été isolés à Sidi Yahia (région d'Oujda) en octobre 1986. Leur conservation est assurée en tubes à essais sur des fragments de tiges de luzerne autoclavés, ce qui permet d'éviter les repiquages successifs sur des milieux synthétiques; on espère ainsi maintenir à son niveau d'origine le pouvoir pathogène. Trois autres isolats sont également d'origine méditerranéenne : du Portugal (région d'Elvas), de Yougoslavie (région de Novi Sad) et de France (Hérault, isolat 34.1). En vue d'établir une comparaison, un isolat de référence d'Eure-etLoir a été utilisé (isolat 28.5). Avant chaque expérimentation, les isolats sont ensemencés sur un milieu nutritif gélosé, à base de farine de maïs à Grignon (Raynal, 1983) et de PSA à Oujda. Les boîtes de Pétri sont mises à incuber à $25^{\circ} \mathrm{C}, 15$ jours dans le premier laboratoire sous lumière continue et 10 jours dans le second, sous un éclairement de 16 heures/jour.

\section{Contamination et notation}

Les plantes âgées d'un mois sont inoculées avec une suspension de spores dosée à 500000 spores $/ \mathrm{ml}$, selon la technique décrite par Raynal (1977) et placées sous une housse en matière plastique transparente permettant de maintenir une humidité relative de $100 \%$. Elles sont éclairées 12 heures/jour à Grignon, 16 heures/jour à Oujda. La température moyenne est de $25^{\circ} \mathrm{C}$, obtenue à Grignon en chambre thermostatée et variant à Oujda dans la salle climatisée réservée aux essais entre $20^{\circ} \mathrm{C}$ (température nocturne) et $30^{\circ} \mathrm{C}$ (température diurne). L'inoculation s'effectue dans ce cas au début de la période d'obscurité, soit en fin d'après-midi.

La notation est faite 11 jours après l'inoculation. L'échelle choisie est celle retenue par Raynal (1977) pour $C$. trifolii. La note 1 correspond à l'absence de symptômes et la note 2 à des taches d'hypersensibilité; les plantes ainsi notées sont considérées comme résistantes. En revanche, les notes 3,4 et 5 sont attribuées à des plantes de plus en plus sensibles, la note 5 caractérisant les plantes mortes.

\section{Résultats}

Résistance des cultivars et lignées de luzerne vis-à-vis des deux isolats marocains de Sidi Yahia

Dans un premier temps, nous avons testé les deux isolats marocains sur l'ensemble des cultivars et lignées. Le Tableau I regroupe les résultats des inoculations effectuées avec l'isolat $n^{\circ} 1$ à Oujda et l'isolat $\mathrm{n}^{\circ} 2$ à Grignon, selon l'origine géographique des luzernes (américaine, flamande, méditerranéenne et australienne). Les résultats sont obtenus en chaque laboratoire sur 4 répétitions de 50 plantes par couple cultivar (ou lignée) - isolat. Notons l'importance de la température sur la réussite des contaminations. Ainsi, une série d'essais préliminaires menés à Oujda a 
Tableau I. Résistance des cultivars de luzerne vis-à-vis de 2 isolats marocains de C. trifolii.

\begin{tabular}{|c|c|c|c|c|c|c|c|c|c|}
\hline & \multirow[t]{2}{*}{ Cultivar ou lignée } & \multicolumn{4}{|c|}{$\begin{array}{l}\text { Pourcentage moyen de plantes résistantes } \\
(\text { notes }(1+2))\end{array}$} & \multicolumn{4}{|c|}{ Note moyenne des symptômes (de 1 à 5) } \\
\hline & & \multicolumn{2}{|c|}{$\begin{array}{l}\text { Isolat n'1 } \\
\text { testé à Oujda }\end{array}$} & \multirow{2}{*}{$\begin{array}{l}\text { Isolat n² } \\
\text { testé à Grignon }\end{array}$} & \multirow{2}{*}{$\begin{array}{l}\text { Moyennes et } \\
\text { groupes } \\
\text { homogènes }\end{array}$} & \multicolumn{2}{|c|}{$\begin{array}{l}\text { Isolat n̊1 } \\
\text { testé à Oujda }\end{array}$} & \multirow{2}{*}{$\begin{array}{l}\text { Isolat n² } \\
\text { testé à Grignon } \\
2,6\end{array}$} & \multirow{2}{*}{$\begin{array}{l}\begin{array}{l}\text { Moyennes et } \\
\text { groupes } \\
\text { homogènes }\end{array} \\
2,2 \mathrm{~F}\end{array}$} \\
\hline & Arc & 78,0 & & & & 1,9 & * & & \\
\hline & MSA CW3 An3 & 62,0 & * & 85,3 & $73,7 \mathrm{~A}$ & 2,4 & & 2,4 & $2,4 \mathrm{E}$ \\
\hline & Beltsville 2An4 & 45,2 & & 52,7 & $49,0 \mathrm{~B}$ & 3,1 & * & 3,3 & $3,2 \mathrm{D}$ \\
\hline Cultivars & Vertus & 17,2 & & 21,1 & $19,2 \mathrm{C}$ & 4,2 & & 4,3 & $4,3 \mathrm{C}$ \\
\hline et lignées & Moapa & 5,0 & & 11,2 & $8,1 \mathrm{D}$ & 4,7 & & 4,5 & $4,6 \mathrm{~B}$ \\
\hline testés & Europe & 3,0 & & 3,8 & $3,4 \mathrm{D}$ & 4,8 & & 4,9 & $4,8 \mathrm{~A}$ \\
\hline à la fois & Magali & 0,0 & & 2,3 & $1,1 \mathrm{D}$ & 5,0 & & 4,9 & $5,0 \mathrm{~A}$ \\
\hline à Oujda & Demnate 4 & 1,5 & & 0,5 & $1,0 \mathrm{D}$ & 4,9 & & 4,9 & $4,9 \mathrm{~A}$ \\
\hline et à & Ziz 8 & 0,0 & & 1,3 & $0,6 \mathrm{D}$ & 5,0 & & 4,9 & $4,9 \mathrm{~A}$ \\
\hline \multirow[t]{6}{*}{ Grignon } & Demnate 1 & 0,5 & & 1,0 & $0,7 \mathrm{D}$ & 4,9 & & 4,9 & $4,9 \mathrm{~A}$ \\
\hline & Mireille & 0,5 & & 0,5 & $0,5 \mathrm{D}$ & 4,9 & & 4,9 & $4,9 \mathrm{~A}$ \\
\hline & Ziz 7 & 0,0 & & 0,0 & $0,0 \mathrm{D}$ & 5,0 & & 4,9 & $4,9 \mathrm{~A}$ \\
\hline & Ziz 10 & 0,0 & & 0,0 & $0,0 \mathrm{D}$ & 4,9 & & 4,9 & $4,9 \mathrm{~A}$ \\
\hline & Moyennes & 16,3 & * & 20,0 & (c.v. $=24 \%$ ) & 4,3 & & 4,3 & (c.v. $=3 \%$ ) \\
\hline & Gémini & 10,8 & & & & 4,4 & & & \\
\hline Cultivars & CAB $77-78$ & 3,1 & & & & 4,8 & & & \\
\hline et lignées & UC Salton & 0,8 & & & & 4,9 & & & \\
\hline testés & Sonora & 0 & & & & 4,9 & & & \\
\hline soit à & WL 514 & 0 & & & & 5,0 & & & \\
\hline \multirow{4}{*}{$\begin{array}{l}\text { Oujda, } \\
\text { soit à } \\
\text { Grignon }\end{array}$} & WL 515 & 0 & & & & 5,0 & & & \\
\hline & Siriver & 0 & & & & 5,0 & & & \\
\hline & MAM M1 C3 & & & 66,6 & & & & 2,6 & \\
\hline & MAP C3 & & & 28,0 & & & & 3,9 & \\
\hline
\end{tabular}

Classement des moyennes (groupes homogènes) par la méthode de Newman-Keuls pour $P=0,01$ (" écart significatif)

échoué, les contaminations ayant été faites le matin et non en soirée. De ce fait, les spores ont été rapidement soumises à une température de $30^{\circ} \mathrm{C}$ dans la salle non thermostatée et les gouttelettes d'inoculum se sont desséchées. Or, les études menées en serre par Welty et Rawlings (1980) montrent qu'une température supérieure à $27^{\circ} \mathrm{C}$ inhibe la formation des appressoria, donc la possibilité de contamination des luzernes. Ces observations nous ont incités à tenir le plus grand compte de ces facteurs dans les essais menés à Oujda sur l'évaluation de la résistance variétale.

Pour les tests effectués à la fois à Oujda et à Grignon, l'analyse de variance montre que les notes et les pourcentages de plantes résistantes offrent une assez bonne concordance, malgré la différence de densité des plantes et les méthodes légèrement dissemblables. On détecte une interaction laboratoire $\mathrm{x}$ cultivar sur les deux variables : pourcentage de plantes résistantes et note moyenne, lesquelles présentent une corrélation linéaire négative ( $r=-0,98$ pour 102 d.I.). L'interaction ne fait cependant que nuancer l'effet prédominant des cultivars $(P<0,001)$; elle s'explique par de rares distorsions entre les résultats des deux laboratoires. Celles-ci, au nombre de 3, correspondent au cas de MSA pour le pourcentage et aux cas d'Arc et Beltsville 2 An4 pour la note; elles ne perturbent pas sensiblement le classement des cultivars. On constate pour les autres cultivars une bonne concordance entre les résultats des deux laboratoires et les notes moyennes sont tout à fait identiques. L'écart entre les pourcentages moyens estimés à Grignon et à Oujda résulte de l'interaction signalée ci-dessus.

Le classement des moyennes des cultivars sur les notes et sur les pourcentages met en évidence une gamme de résistance, avec par ordre décroissant Arc, MSA, Beltsville 2An4, Vertus, Moapa et le groupe homogène des 8 autres cultivars.

Pour les autres tests, effectués soit à Oujda ( 8 cultivars), soit à Grignon (2 lignées), on constate que les cultivars ou lignées ont une résistance très faible à nulle, sauf les lignées de type méditerranéen MAM M1 C3 et MAP C3 qui ont subi trois cycles de sélection à l'anthracnose à la Station d'Amélioration des Plantes INRA de Montpellier. Il en est de même pour le cultivar Gémini qui, avec un niveau de résistance en conditions 
artificielles, légèrement supérieur à $10 \%$, s'avère assez résistant au champ (Anonyme, Bulletin des variétés, 1984).

Les cultivars et lignées de $M$. sativa se répartissent donc en 3 groupes :

- résistants (plus de $50 \%$ de plantes résistantes): Arc, MSA CW3 An3, Beltsville 2An4, MAM M1 C3;

- assez résistants (10 à $30 \%$ de plantes résistantes) : MAP C3, Vertus, Gémini);

- sensibles à très sensibles $(0$ à $10 \%$ de plantes résistantes) : Moapa, Europe, CAB 77-78, Magali, Mireille, UC Salton, Sonora, WL 514 et 515, Siriver, Demnate 1 et 4, Ziz 7, 8, 10.

Le classement des cultivars d'origine américaine et flamande par niveau de résistance face aux deux isolats marocains, est en tous points similaire, malgré des méthodes de contamination et de notation différentes, à celui établi d'après les résultats publiés par divers chercheurs : en Europe par Gondran et Mainer Casado (1973), Raynal (1977), Gondran (1982) et en Amérique par Devine et al. (1975) et Ostazeski et al. (1979).

\section{Pouvoir pathogène d'isolats d'origines géogra- phiques différentes}

A partir de 1978, la diffusion aux U.S.A. de cultivars résistants a favorisé l'apparition de mutants virulents de $C$. trifolii, capables de surmonter les gènes de résistance de l'hôte (Ostazeski et al., 1979; Welty et Mueller, 1979; Allen et al., 1982; Elgin et Ostazeski, 1982; Welty et al., 1982). L'apparition aux Etats-Unis de races virulentes du pathogène nous a amenés à amorcer l'étude de la variabilité des populations de $C$. trifolii en confrontant $M$. truncatula et des cultivars de $M$. sativa de diverses origines à cinq isolats du pathogène (quatre de pays méditerranéens et un de référence, C.28.5, dont nous connaissions l'effet sur la plupart des cultivars testés; Raynal, 1977) (Tableau II).

Pour ce test, les contaminations ont été effectuées en chambre climatisée à Grignon sur 100 plantes par combinaison cultivar $\mathrm{x}$ isolat, réparties en deux blocs (expériences décalées dans le temps).

La variabilité intracultivar diffère selon les cultivars, tant pour les pourcentages de plantes résistantes que pour les notes de symptômes. Pour ces deux variables, les écarts-types sont supérieurs chez les deux cultivars américains, qui par ailleurs présentent le plus de plantes résistantes. Au contraire, l'écart-type de Magali, cultivar le plus sensible, est significativement moindre.

En ce qui concerne les isolats, seule la variabilité intra-isolat des notes de symptômes est hétérogène, elle est plus faible pour "Sidi Yahia" et «Portugal».

$M$. truncatula que nous savions totalement résistante à l'isolat C.28.5 (Raynal, 1977) s'avère totalement résistante vis-à-vis des quatre autres isolats. Les résultats, complètement homogènes, n'ont pas été pris en compte dans l'analyse de variance.

Après transformation angulaire de BLISS sur les pourcentages de plantes résistantes et malgré un coefficient de variation élevé (c.v. $=24 \%$ ), on met en évidence (Tableau Ila), en l'absence d'interaction, des effets significatifs du cultivar et de l'isolat (resp. à $P=1 \%$ et $5 \%$ ). Le classement des valeurs moyennes discrimine bien les cultivars : depuis Arc, le plus résistant, jusqu'à Magali, le plus sensible, il apparaît un groupe homogène constitué par Europe, Demnate 1, Ziz 10 et Mireille. Quant aux isolats, on constate les moindres effets de "28.5» et de «34.1" par rapport à ceux de "Portugal».

Les notes moyennes des symptômes (Tableau IIb) sont en corrélation linéaire négative avec la variable précédente $(r=-0,995$ pour 68 d.I.). Leur variabilité est plus faible (c.v. $=12 \%$ ). Elles font l'objet d'une interaction cultivar $\mathrm{x}$ isolat, mais l'effet très largement prédominant est celui des cultivars. Leur classement corrobore celui obtenu pour les pourcentages, en distinguant, par ordre de sensibilité croissante : Arc, Moapa et le groupe des 5 autres cultivars.

L'interaction mise en évidence est due à la sensibilité différentielle des cultivars Arc et Moapa à certains isolats. En effet, alors que toutes les notes moyennes des combinaisons mettant en jeu les 5 autres cultivars sont statistiquement homogènes (puissance a posteriori = $99 \%)$, Arc et Moapa répondent de façon différente aux 5 isolats. La représentation graphique de ce phénomène d'après les données du Tableau Ilb (Fig. 1) montre que pour Arc, l'intensité des symptômes va en croissant selon l'ordre suivant des isolats : 28.5, Portugal, Sidi Yahia, 34.1, Yougoslavie. Pour Moapa, l'ordre est différent : 28.5, 34.1 , Yougoslavie, Sidi Yahia, Portugal. Pour tous les autres cultivars, par contre, l'intensité des symptômes est identique quel que soit l'isolat.

La signification biologique de cette interaction est difficile à préciser. Elle pourrait indiquer que les isolats appartiennent à des races montrant des virulences différentes sur Arc et sur Moapa. Cependant, le niveau d'agressivité très élevé de l'ensemble des isolats sur Moapa tend à diminuer la netteté et la portée du phénomène. Une autre hypothèse, tout aussi vraisemblable, est simplement que la variation interne des essais 
Tableau II. Pouvoir pathogène de 5 isolats de $C$. trifolii vis-à-vis de cultivars de luzerne de diverses origines et d'une luzerne sauvage.

a) Pourcentages moyens de plantes résistantes (notes $1+2$ ).

Cultivars

Isolats

\begin{tabular}{|c|c|c|c|c|c|}
\hline 28.5 & 34.1 & Sidi Yahia & & & Moyennes \\
\hline France & France & Maroc & & & $\begin{array}{l}\text { homogènes } \\
(P=0,01)\end{array}$ \\
\hline
\end{tabular}

\begin{tabular}{|c|c|c|c|c|c|c|c|c|}
\hline \multirow{7}{*}{$\begin{array}{l}\text { Medicago } \\
\text { sativa }\end{array}$} & Arc & 82,0 & 71,4 & 71,7 & 71,1 & 71,5 & $73,5 \mathrm{~A}$ & 2,9 \\
\hline & Moapa & 12,9 & 11,0 & 7,1 & 6,3 & 11,0 & $9,7 \mathrm{~B}$ & 2,5 \\
\hline & Europe & 4,0 & 6,0 & 2,1 & 1,0 & 3,1 & $3,2 \mathrm{C}$ & 1,8 \\
\hline & Demnate 1 & 4,0 & 1,0 & 2,1 & 2,1 & 3,2 & $2,5 \mathrm{C}$ & 1,2 \\
\hline & Ziz 10 & 3,1 & 2,4 & 2,4 & 1,0 & 2,3 & $2,2 \mathrm{C}$ & 1,5 \\
\hline & Mireille & 2,0 & 7,1 & 0 & 0 & 1,0 & $2,0 \mathrm{C}$ & 1,4 \\
\hline & Magali & 0 & 0 & 0 & 0 & 0 & $0 \quad D$ & 0,4 \\
\hline \multirow{2}{*}{\multicolumn{2}{|c|}{$\begin{array}{l}\text { Moyennes et groupes } \\
\text { homogènes * } \\
(P=0,05) \\
\text { Medicago truncatula * }\end{array}$}} & $\begin{array}{l}15,4 \\
A\end{array}$ & $\begin{array}{l}14,1 \\
A\end{array}$ & $\begin{array}{l}12,2 \\
A B\end{array}$ & $\begin{array}{l}11,6 \\
B\end{array}$ & $\begin{array}{l}13,1 \\
A B\end{array}$ & & \\
\hline & & 100 & 100 & 100 & 100 & 100 & 100 & \\
\hline
\end{tabular}

b) Note moyenne des symptômes (de 1 à 5)

Moyennes
et groupes
homogènes
$(P<0,001)$

\begin{tabular}{|c|c|c|c|c|c|c|c|c|}
\hline & Arc & $1,95 \mathrm{f}$ & $2,62 d c$ & $2,59 \mathrm{dc}$ & $2,40 \mathrm{e}$ & $2,81 \mathrm{~d}$ & $2,47 \mathrm{C}$ & 0,09 \\
\hline & Moapa & $4,42 \mathrm{c}$ & $4,49 b c$ & $4,68 a b$ & $4,80 \mathrm{a}$ & $4,50 \mathrm{bc}$ & $4,58 \mathrm{~B}$ & 0,09 \\
\hline & Europe & 4,78 a & 4,79 a & 4,91 a & $4,88 a$ & 4,93 a & $4,86 \mathrm{~A}$ & 0,06 \\
\hline Medicago & Demnate 1 & $4,82 a$ & 4,96 a & $4,94 \mathrm{a}$ & 4,85 a & 4,86 a & $4,89 \mathrm{~A}$ & 0,05 \\
\hline \multirow[t]{4}{*}{ sativa } & $\mathrm{Ziz} 10$ & $4,85 \mathrm{a}$ & 4,91 a & $4,90 \mathrm{a}$ & 4,91 a & $4,90 \mathrm{a}$ & $4,91 \mathrm{~A}$ & 0,07 \\
\hline & Mireille & 4,93 a & $4,78 \mathrm{a}$ & $5 a$ & $5 a$ & 4,93 a & $4,93 \mathrm{~A}$ & 0,04 \\
\hline & Magali & 5 a & 4,97 a & $5 a$ & $5 a$ & 5 a & $4,99 \mathrm{~A}$ & 0,01 \\
\hline & e.t. (3) & 0,09 & 0,07 & 0,04 & 0,04 & 0,07 & & \\
\hline \multicolumn{2}{|c|}{ Medicago truncatula * } & 1,00 & 1,00 & 1,00 & 1,00 & 1,00 & 1,00 & \\
\hline
\end{tabular}

\footnotetext{
- Groupes homogènes de Newman-Keuls

- données analysées après transformation angulaire

- e.t. (écart-type «intra») : (1) $S$ à $P=10^{-4}$

(2) $S$ à $P=10^{-3}$

_** Hors analyse statistique

(3) $S$ a $P=7 \%$
}

est la cause de cette interaction. II est donc probable que les isolats testés ne se distinguent pas clairement par des caractères de virulence, alors qu'ils sont très comparables en ce qui concerne leur agressivité, au moins sur les cultivars utilisés. Néanmoins, l'existence possible de races, résultant de la grande aptitude à varier que possèdent les Colletotrichum, doit nous mettre en garde contre une mauvaise utilisation des gènes de résistance.

\section{Conclusion}

Si des conditions climatiques et culturales (irrigation) particulièrement favorables se trouvent réunies, on peut craindre qu'une épidémie d'anthracnose ne se généralise dans le Maroc oriental. Pour prévenir cette éventualité, on pourrait envisager la culture des cultivars américains résistants de $M$. sativa, ou le développement de cultivars de type méditerranéen à partir de 


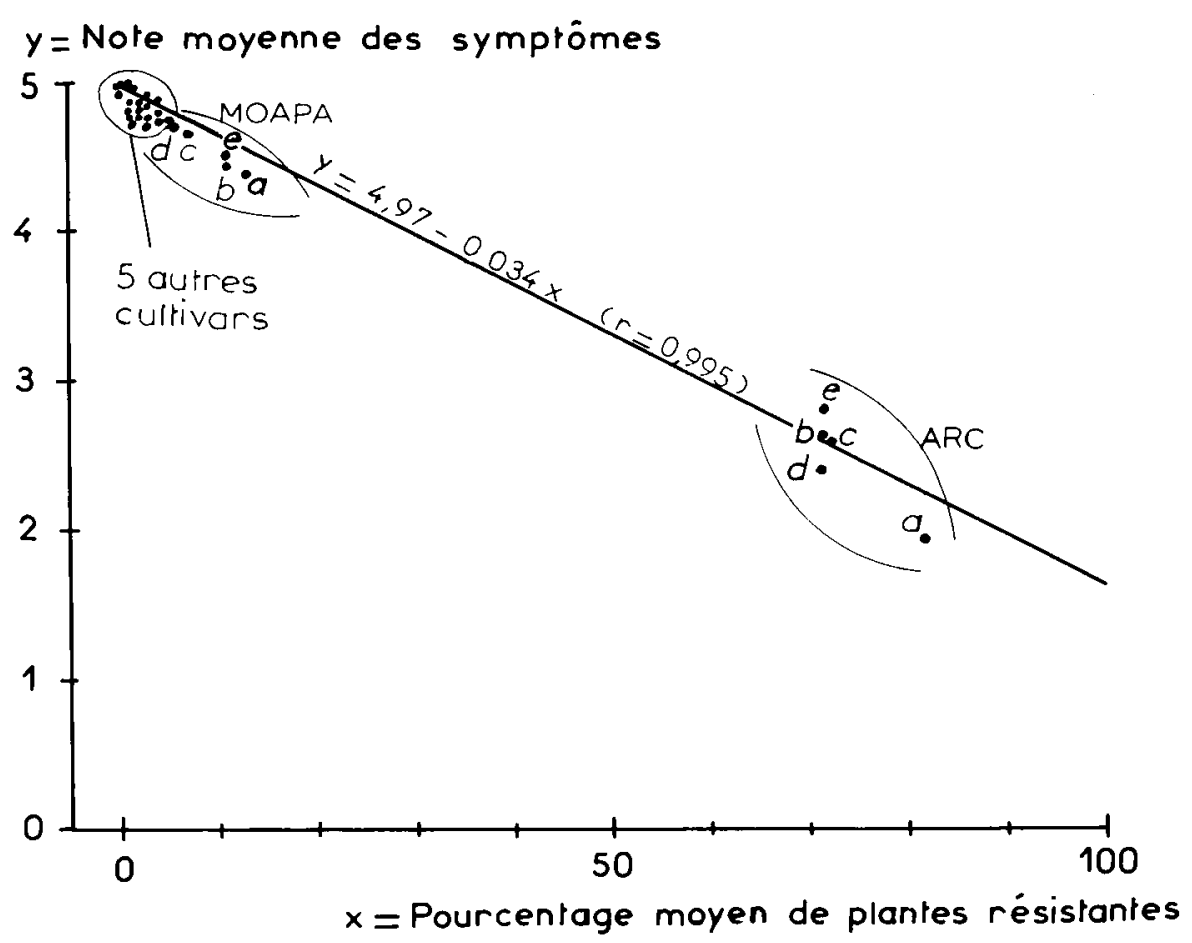

Fig. 1. Relation entre pourcentage moyen de plantes de luzerne résistantes à $C$. trifolii et note moyenne des symptômes, pour 7 cultivars testés vis-à-vis des 5 isolats suivants : $a$ : 28.5; $b: 34.1$; $:$ Sidi Yahia $n^{\circ} 2$; $d$ : Portugal; e : Yougoslavie.

lignées à bonne résistance (comme MAN M1 C3, MAP C3, sélectionnées par l'INRA à Montpellier), ou l'introduction dans $M$. sativa de résistances à partir de Medicago sauvages, pérennes, cette dernière proposition étant la plus délicate.

Pour l'instant, les isolats marocains testés semblent appartenir à la race 1 de $C$. trifolii, telle que l'ont définie Elgin et Ostazeski (1982), la plus commune dans le monde, de même que les autres isolats méditerranéens et l'isolat du Nord de la France. Cette race est celle qui prédomine lorsqu'aucun cultivar possédant une résistance de type monogénique n'a effectué de pression de sélection sur la population parasitaire. Contre cette race, les cultivars et lignées américains résistants ainsi qu'une lignée française, sélectionnés en utilisant d'autres isolats de la race 1, s'avèrent efficaces. Toutefois, le risque est grand de voir apparaître comme aux Etats-Unis des races de virulences nouvelles capables de surmonter les résistances monogéniques lorsque les cultivars les possédant seront largement diffusés.

En ce qui concerne les cultivars européens moyennement résistants que nous avons testés, Vertus et Gémini ont une résistance intéressante, mais appartiennent au type flamand. En vue d'estimer leur capacité d'adaptation aux conditions climatiques du Maroc oriental, nous avons mis en place quelques parcelles d'essais chez un agriculteur. Pour ce qui est du cultivar Moapa, reconnu pour ses bonnes qualités agronomiques et son adaptation au climat régional, nous pouvons espérer améliorer son niveau de résistance après 2 ou 3 cycles de sélection, ce que nous avons entrepris. En effet, l'étude réalisée par Guy (1976) laisse supposer qu'un seul gène majeur intervient (même si, d'après cet auteur comm. pers. -, il semblerait exister plusieurs résistances différentes), d'où la rapidité espérée de la réponse de l'hôte à la sélection. Les écotypes locaux, fortement sensibles à l'anthracnose, devraient être améliorés vis-à-vis de cette maladie, car ils montrent par ailleurs un intérêt certain. La sélection massale intra-familiale semble la plus susceptible de conduire à une amélioration rapide de la résistance de la luzerne à l'anthracnose, tout en sachant que l'on doit surveiller l'apparition éventuelle de races virulentes de C. trifolii.

\section{Remerciements}

Les auteurs remercient Messieurs A. Birouk (IAV Hassan II, Rabat) et M. Angevain (INRA, Montpellier) pour la fourniture de semences et divers renseignements concernant les cultivars ou lignées de luzerne.

\section{Références}

Anonyme (1984) Bulletin des Variétés, Plantes fourragères pérennes. INRA-GEVES

Allen S.J., Barnes G.L. \& Caddel J.L. (1982) A new race of Colletotrichum trifolii on alfalfa in Oklahoma. Plant. Dis. 66 (10), 922-924 
Arnon D.I. \& Hoagland D.R. (1940) Crop production in artificial culture solutions and soils with special reference to factors influencing yield and absorption of inorganic nutrients. Soil Sci., 50, 463-483

Bain S.M. \& Essary S.H. (1906) A new anthracnose of alfalfa and red clover. J. Mycol., 12, 192-193

Barnes D.K., Ostazeski S.A., Schillinger J.A. \& Hanson C.H. (1969) Effect of anthracnose (Colletotrichum trifolii ) infection on yield, stand and vigor of alfalfa. Crop Sci., 9, 344-346

Devine T.E., Campell T.A. \& Hanson C.H. (1975) Anthracnose disease ratings for alfalfa varieties and experimental strains. Technic. Bull., 1507, ARS-USDA

Elgin J.H. et al. (19 co-auteurs) (1981) Anthracnose resistance increases alfalfa yields. Crop Sci., 21, 457460

Elgin J.H. \& Ostazeski S.A. (1982) Evaluation of selected alfalfa cultivars and related Medicago species for resistance to race 1 and race 2 of anthracnose. Crop. Sci., 22, 39-42

Gondran J. (1982) Résistance à l'anthracnose des cultivars de luzerne inscrits au Catalogue français. Fourrages, 91, 99-103

Gondran J. \& Mainer Casado A. (1973) Inoculation artificielle de la luzerne avec Colletotrichum trifolii Bain et Essary. Classement de 19 cultivars de luzerne suivant leur résistance au parasite. Ann. Amélior. Plantes, 23 (4), 367-379
Guy P. (1976) Déterminisme génétique de la résistance à Colletotrichum trifolii chez Medicago sativa $\mathrm{L}$. Ann. Amélior. Plantes, 26 (2), 215-234

Ostazeski S.A., Elgin J.H. Jr. \& Mc Murtrey J.E. (1979) Occurence of anthracnose on formerly anthracnoseresistant "Arc" alfalfa. Plant Dis. Rept., 63 (9), 734-736

Raynal G. (1977) Comparaison, en contaminations artificielles, des pouvoirs pathogènes des Colletotrichum isolés en France sur la luzerne. Ann. Phytopathol., 9 (2), 193-203

Raynal G. (1983) Contribution à l'étude des mycoses de la luzerne et du trèfle violet. Cas de trois maladies nécrotiques : anthracnose, pepper-spot, sclérotiniose. Possibilités de lutte. Thèse Doctorat ès Sci., Fac. Sci. Orsay

Trœung B.M. \& Gosset H. (1987) Première observation de l'anthracnose de la luzerne dans le Maroc Oriental. Agronomie, 7 (5), 361-363

Welty R.E. \& Mueller J.P. (1979) Occurrence of a highly virulent isolate of Colletotrichum trifolii on alfalfa in North Carolina. Plant Dis. Rept., 63, 666-670

Welty R.E. \& Rawlings J.O. (1980) Effects of temperature and light on development of anthracnose on alfalfa. Plant Dis., 64, 476-478

Welty R.E., Gurgis R.Y. \& Rowe D.E. (1982) Occurrence of race 2 of Colletotrichum trifolii in North Carolina and resistance of alfalfa cultivars and breeding lines to races 1 and 2. Plant Dis., 66, 48-51 